\title{
ANÁLISE FÍSICO-QUÍMICA E SENSORIAL DE PÃO FRANCÊS COM REDUÇÃo DE SÓDIO E ENRIQUECIDO COM FIBRAS
}

\author{
Krischina Singer APLEVICZ ${ }^{1}$ \\ Simone Delezuk INGLEZ ${ }^{2}$ \\ Eduardo Sidinei CHAVES ${ }^{3}$ \\ Mariana MARTELLI ${ }^{4}$ \\ Bruno Luís FERREIRA ${ }^{5}$
}

\begin{abstract}
${ }^{1}$ Doutora em Ciências dos Alimentos, Professora do Instituto Federal de Santa Catarina, Campus Florianópolis-Continente, aplevicz@gmail.com

${ }^{2}$ Doutora em Ciências, Professora Universidade Tecnológica Federal do Paraná, Campus Ponta Grossa- Paraná, simoneinglez@utfpr.edu.br

${ }^{3}$ Doutor em Química Analítica, Professor na Universidade Tecnológica Federal do Paraná, Campus Ponta Grossa- Paraná, eschaves@utfpr.edu.br

${ }^{4}$ Mestre em Turismo, Professora do Instituto Federal de Santa Catarina, Campus FlorianópolisContinente, mariana.martelli@ifsc.edu.br

${ }^{5}$ Bolsista PIBIC da Fundação Araucária, da Universidade Tecnológica Federal do Paraná, Campus Ponta Grossa-Paraná, bru.c8@ hotmail.com
\end{abstract}

Recebido em: 30/05/2013 - Aprovado em: 14/11/2014 - Disponibilizado em: 15/12/2014

RESUMO: O pão francês é o produto de panificação mais consumido no Brasil, preparado basicamente com farinha de trigo, água, sal e melhorador. O objetivo desse estudo foi analisar fisico-quimicamente e sensorialmente formulações de pão francês enriquecido de fibras e com teor reduzido de sal. Os pães foram submetidos a análise de umidade, cinzas, proteína, lipídeos, fibra bruta, sódio e potássio. A análise sensorial de aceitabilidade foi aplicada utilizando-se a escala hedônica de nove pontos, com julgadores não treinados. A amostra $\mathrm{C}$ apresentou o maior teor de fibras e potássio e menor teor de sódio $(\mathrm{P}<0,05)$. Todas as amostras foram aceitas, sendo a amostra $\mathrm{C}$, com farelo de trigo a de maior aceitabilidade.

Palavras-chave: pão francês, sódio, fibras.

\section{PHYSICO-CHEMICAL AND SENSORY EVALUATION OF BREAD FRENCH WITH REDUCED SODIUM RICH AND FIBER}

\begin{abstract}
The french bread is the bakery product is mostly consumed in Brazil, basically prepared with wheat flour, water, salt and improver. The aim of this study was to analyze physicochemical and sensory formulations enriched French bread with fiber and low salt content. The breads were subjected to analysis of moisture, ash, protein, lipid, crude fiber, sodium and potassium. The sensory acceptability analysis was applied using the nine-point hedonic scale, with no trained judges. Sample $\mathrm{C}$ had the highest content of fiber and potassium and lower sodium content $(\mathrm{P}<0.05)$. All samples were accepted, and the sample $\mathrm{C}$, with wheat bran to greater acceptability.

Keywords: french bread, sodium, fiber.
\end{abstract}




\section{INTRODUÇÃO}

Pão é um dos principais alimentos básicos consumido diariamente em todas as partes do mundo. Embora exista uma grande variedade de diferentes tipos, o termo pão geralmente se refere a produtos fermentados que contenham trigo (HAGER et al., 2012).

O pão francês é o resultado do cozimento da massa feita com farinha de trigo, água, sal e fermento biológico, considerados ingredientes básicos para sua produção (ORNELLAS, 2001)

Segundo dados da Associação Brasileira da Indústria de Panificaçãoe Confeitaria (ABIP, 2014) O consumo per capita do pão francês é de aproximadamente $56 \mathrm{~g}$ por dia, o que equivale o consumo de pelo menos um pão por dia.

Por ser o pão mais consumido no Brasil, o Ministério da Saúde, a Associação Brasileira das Indústrias de Alimentação (ABIA), Associação Brasileira das Indústrias de Massas Alimentícias (ABIMA), Associação Brasileira da Indústria de Trigo (ABITRIGO) e a Associação Brasileira da Indústria de Panificação e Confeitaria (ABIP) firmaram no ano de 2011 um termo de compromisso visando reduzir o índice de sal neste produto até o final de 2014 (MINISTÉRIO DA SAÚDE, 2011). O consumo diário de sal no Brasil é de $12 \mathrm{~g}$ per capita, sendo $5 \mathrm{~g}$ o recomendado pela
Organização Mundial de Saúde (ANVISA, 2012).

O pão francês é um produto de consumo diário do brasileiro que apresenta elevadas quantidades de sódio (PHILIPPI et al., 1996). As principais funções tecnológicas do sal em produtos de panificação são o favorecimento do desenvolvimento da rede de glúten e o reforço das propriedades viscoelásticas da massa, assim como a inibição da atividade da levedura na fermentação e o controle da atividade de água no pão cozido (CAUVAIN, 2007). Porém, consumir excessivamente o sódio faz com que ocorra a liberação de alguns hormônios que causam a retenção de líquidos, aumentando a pressão arterial (MENDONÇA e SILVA, 2012).

As fibras alimentares têm demonstrado benefícios à manutenção da saúde e prevenção de doenças. Estão presentes nos componentes de dietas ricas em carboidratos e são classificadas como fibras solúveis e insolúveis. São vários os benefícios atribuídos ao consumo adequado de fibras alimentares, verificados através de estudos clínicos e epidemiológicos, como por exemplo: diminuição do colesterol; redução de risco e controle de doenças como a obesidade, doenças cardiovasculares e diabetes (ADA, 2002).

Tendo em vista a crescente importância econômica do setor da 
panificação e a exigência legal de produtos com redução do teor de sal, visando a prevenção da saúde, o objetivo desse estudo foi analisar fisico-quimicamente $\mathrm{e}$ sensorialmente formulações de pão francês enriquecido de fibras e com teor reduzido de sal.

\section{MATERIAIS E MÉTODOS}

\section{Elaboração dos pães}

A formulação dos pães foi baseada no guia de boas práticas nutricionais do pão francês [6] e está ilustrada na Tabela 1. Os pães foram elaborados a partir do método direto, adicionando os ingredientes secos e, em seguida, a água a $5{ }^{\circ} \mathrm{C}$. A massa foi misturada em masseira rápida (marca Líder) por 5 minutos e submetida a um descanso de mesa de 30 minutos. Em seguida, foi dividida em porções de $70 \mathrm{~g}$, boleadas, passadas em modeladora (marca Líder). Por fim, os pães foram fermentados por $2 \mathrm{~h}$ em câmara de fermentação controlada (marca Perfecta) a 30 ${ }^{\circ} \mathrm{C}$ (80\% umidade relativa). $\mathrm{O}$ assamento foi realizado com vapor em forno turbo (marca Lider) a $200{ }^{\circ} \mathrm{C}$ por $10 \mathrm{~min}$. Os pães foram elaborados no Laboratório de Panificação do Instituto Federal de Santa Catarina, Campus Florianópólis-Continente.

Tabela 1 - Formulação dos pães tipo francês

\begin{tabular}{llll}
\hline Ingredientes (\%) & A & B & C \\
\hline Farinha de trigo & 100 & 100 & 95 \\
Farelo de trigo & - & - & 5 \\
Água & 60 & 60 & 60 \\
Sal & 2 & 1,8 & 1,8 \\
Fermento biológico seco & 2 & 2 & 2 \\
Melhorador & 1 & 1 & 0,8 \\
A: Formulação padrão utilizada hoje; & & \\
B: Formulação recomendada pela ANVISA até o ano de 2014; & & \\
C: Formulação recomendada pela ANVISA até o ano de 2014, com adição de farelo de trigo e redução de \\
melhorador.
\end{tabular}

\section{Análises físico-química}

As análises das amostras dos pães tipo francês foram realizadas segundo os métodos estabelecidos pelo Instituto Adolfo Lutz (1985). Os parâmetros analisados foram: umidade, cinzas, proteína, lipídeos, fibra bruta, sódio e potássio. As análises foram realizadas no Laboratório de Métodos Instrumentais da Universidade Tecnológica Federal do Paraná, Campus de Ponta Grossa. 


\section{Volume específico e perda de umidade}

O volume dos pães foi medido usando a metodologia de deslocamento de sementes (HALLÉN et al., 2004). O volume do pão foi medido em um recipiente com volume conhecido $\left(\mathrm{V}_{\mathrm{C}}\right)$. $\mathrm{O}$ recipiente foi coberto com sementes, o pão foi removido e o volume de sementes anotado $\left(\mathrm{V}_{\mathrm{R}}\right)$. $\mathrm{O}$ volume do pão (VL) foi calculado seguindo a Equação (1).

$\mathrm{VL}(\mathrm{mL})=\mathrm{VC}-\mathrm{VR}$

Após esfriar $1 \mathrm{~h}$, os mesmos pães foram medidos e pesados em escala digital (g). O volume específico (VS) do pão foi calculado seguindo a Equação (2).

$\mathrm{VS}(\mathrm{mL} / \mathrm{g})=\mathrm{VL} / \mathrm{P}$

A perda de umidade foi medida deduzindo o peso do pão assado do peso inicial da massa antes do assamento (PLESSAS et al., 2007). Os experimentos foram realizados em triplicata.

\section{Análise sensorial}

A análise sensorial foi realizada de acordo com a metodologia descrita por Meilgaard; Civille e Carr (2007). O teste de aceitabilidade foi aplicado a um grupo de julgadores não treinados $(n=50)$, utilizando a escala hedônica estruturada de nove pontos (1 - desgostei extremamente; 9 - gostei extremamente). Foi realizado teste de intenção de compra da amostra elaborada com farelo de trigo e menor quantidade de melhorador, por ter adição de fibras e menor teor de sal. A escala utilizada foi de 5 pontos ( $5=$ certamente compraria, $3=$ indiferente, 1 $=$ certamente não compraria) (DELLA TORRE et al., 2003). Foi aplicado um questionário para se obter informações sobre os julgadores.

\section{Análise estatística}

Os valores obtidos nas análises sensoriais foram avaliados estatisticamente pelo programa Statistica ${ }^{\circledR}$ versão 8.0 (Statsoft Inc., Tulsa, OK, USA). As diferenças entre as médias foram calculadas através da análise de variância (ANOVA) one-way utilizando o teste de Tukey. Foram consideradas significativas as diferenças ao nível de $5 \%$ (P $<0,05)$.

\section{RESULTADOS E DISCUSSÃO}

Os resultados físico-químicos dos pães estão apresentados na Tabela 2. A amostra C apresentou maior teor de umidade $(\mathrm{P}<0,05)$. Este resultado se deve a incorporação da fibra na formulação, favorecendo o aumento da retenção de água no produto final. Isto pode estar relacionado diretamente ao aumento da absorção de água, principalmente devido ao grande número de grupos hidroxila presentes na estrutura das fibras alimentares, permitindo 
maior interação com a água por meio de ligações de hidrogênio (BORGES et al., 2011). Não foi observado diferença significativa entre os teores de proteínas e lipídios $(\mathrm{P}>0,05)$.

A amostra C, com farelo de trigo, foi a que apresentou maior teor de fibras, com 1,03\% (P < 0,05). A utilização de altas concentrações de fibras dietéticas em pães produz modificações nas características da massa e do pão. A fibra aumenta a absorção de água da massa e o tempo de desenvolvimento da mesma e diminui a capacidade de retenção de gás e, consequentemente, o volume do pão (LAI et al., 1989).

O teor de sódio foi menor para a amostra $\mathrm{C}$, que continha teor reduzido de sal e de melhorador. Vieira et al. (2007) determinaram o teor de sódio contido no pães branco e encontraram valores entre 3,44 e $7,18 \mathrm{mg} / \mathrm{g}$. O desenvolvimento de receitas com teor reduzido de sódio é de significativa importância, pois devido ao elevado número de pacientes com restrição de sódio na dieta, serve como instrumento para auxiliar nas orientações nutricionais (SILVA et al., 2003). O sódio é um micronutriente essencial ao organismo e constitui $40 \%$ do sal de cozinha, sendo que $10 \%$ do sódio ingerido apresenta sua origem no conteúdo natural dos alimentos e o restante é proveniente de adição (VIEGAS, 2008). A Agência Nacional de Vigilância Sanitária (ANVISA) propôs a diminuição do sal na formulação do pão francês de $2 \%$ para $1,8 \%$, reduzindo assim em $10 \%$ a quantidade de sal o que equivale a $4 \%$ de sódio (ANVISA, 2012).

Tabela 2 - Análises físico-químicas das amostras de pães tipo francês.

\begin{tabular}{lccc}
\hline \multicolumn{1}{c}{ Constituíntes } & A & B & C \\
\hline Umidade (\%) & $35,98^{\mathrm{a}} \pm 0,15$ & $36,06^{\mathrm{a}} \pm 0,14$ & $37,21^{\mathrm{b}} \pm 0,05$ \\
Cinzas (\%) & $1,85^{\mathrm{a}} \pm 0,04$ & $1,60^{\mathrm{b}} \pm 0,06$ & $1,57^{\mathrm{b}} \pm 0,05$ \\
Proteína (\%) & $10,10^{\mathrm{a}} \pm 0,05$ & $10,70^{\mathrm{a}} \pm 0,23$ & $10,83^{\mathrm{a}} \pm 1,57$ \\
Lipídeos (\%) & $0,77^{\mathrm{a}} \pm 0,10$ & $0,80^{\mathrm{a}} \pm 0,10$ & $0,81^{\mathrm{a}} \pm 0,4$ \\
Fibra bruta (\%) & $0,42^{\mathrm{a}} \pm 0,16$ & $0,70^{\mathrm{a}} \pm 0,08$ & $1,03^{\mathrm{b}} \pm 0,15$ \\
Sódio $(\mathrm{mg} / \mathrm{g})$ & $5,24^{\mathrm{a}} \pm 0,01$ & $5,22^{\mathrm{a}} \pm 0,03$ & $4,94^{\mathrm{b}} \pm 0,07$ \\
Potássio $(\mathrm{mg} / \mathrm{g})$ & $1,37^{\mathrm{a}} \pm 0,03$ & $1,52^{\mathrm{b}} \pm 0,03$ & $1,80^{\mathrm{c}} \pm 0,05$ \\
\hline
\end{tabular}

* Resultados como média \pm desvio padrão de duas replicatas. ${ }^{\text {a-c }}$ Letras diferentes sobrescritas na mesma coluna indicam diferença significativa entre os tratamentos (Teste de Tukey $\mathrm{P}<0,05$ ).

Legenda:

A: Formulação padrão utilizada hoje;

B: Formulação recomendada pela ANVISA até o ano de 2014;

C: Formulação recomendada pela ANVISA até o ano de 2014, com adição de farelo de trigo e redução de melhorador. 
Os resultados das análises de volume e perda de umidade estão apresentados na Tabela 3. Não foi encontrada diferença significativa na análise de perda de umidade, apresentando a maior perda de umidade para a amostra padrão (A). Com relação ao volume, os pães não apresentaram diferença signifitativa nos tratamentos, não tendo a adição de farelo de trigo e redução do melhorador influenciado no volume. $\mathrm{O}$ volume do pão depende da capacidade da massa em reter o gás por um tempo prolongado durante a fermentação e o cozimento (DELCOUR e HOSENEY, 2010). Em panificação, o sal tem função de fixador, fortificante do glúten, fazendo com que retenha o gás produzido pelo fermento, branqueador do miolo dos pães e controlador da fermentação, sendo que sua ausência prejudica o crescimento dos pães (HALLÉN et al., 2004).

Os resultados do teste de aceitabilidade foram analisados pelo teste de Tukey estão ilustrados na Tabela 3. A partir dos valores das médias dos pães obtidas pelo teste de aceitabilidade, foi verificada a ausência de diferença significativa entre as amostras com A, B e C $(\mathrm{P}>0,05)$. A melhor nota sensorial foi para o pão com farelo de trigo na sua formulação e com teor reduzido de sal e melhorador. No teste sensorial, 64\% dos julgadores foram mulheres. A totalidade dos julgadores foi classificada como grandes consumidores de pão, pois $12 \%$ relataram consumir três ou mais vezes ao dia, $36 \%$ duas vezes e $34 \%$ uma vez ao dia. Destes, apenas $38 \%$ tem preferência pelo consumo de pão integral. Na Figura 1 está ilustrado os miolos das amostras dos pães.

Tabela 3 - Resultados de perda de umidade, volume específico e análise sensorial dos pães tipo francês.

\begin{tabular}{cccc}
\hline Amostras & $\begin{array}{c}\text { Perda de umidade } \\
(\mathrm{g})\end{array}$ & $\begin{array}{c}\text { Volume específico } \\
(\mathrm{mL} / \mathrm{g})\end{array}$ & $\begin{array}{c}\text { Análise } \\
\text { sensorial }\end{array}$ \\
\hline A & $6,41 \pm 0,59^{\mathrm{a}}$ & $6,07 \pm 0,67^{\mathrm{a}}$ & $7,06^{\mathrm{a}}$ \\
B & $4,88 \pm 1,11^{\mathrm{a}}$ & $5,85 \pm 0,10^{\mathrm{a}}$ & $6,98^{\mathrm{a}}$ \\
C & $5,36 \pm 0,51^{\mathrm{a}}$ & $5,20 \pm 1,15^{\mathrm{a}}$ & $7,30^{\mathrm{a}}$ \\
\hline
\end{tabular}

Resultado como média \pm desvio padrão. ${ }^{a}$ Letras sobrescritas diferentes na mesma coluna indicam diferença significativa entre os tratamentos (Teste de Tukey, $\mathrm{P}<0,05)$.
A: formulação padrão utilizada hoje;
B: formulação recomendada pela ANVISA até o ano de 2014;
C: formulação recomendada pela ANVISA até o ano de 2014, com adição de farelo de trigo e redução melhorador. 


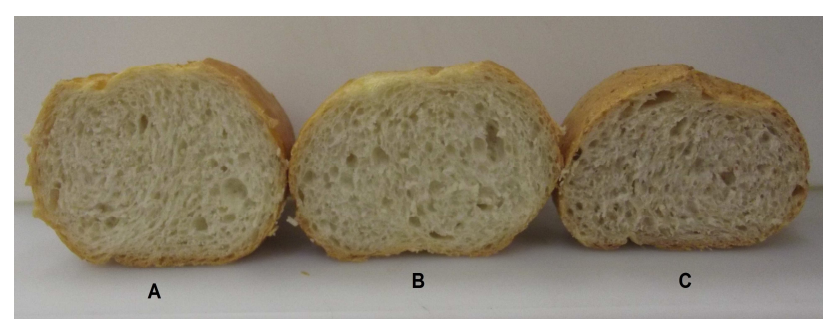

Figura 1. Miolo dos pães tipo francês.

Silva et al. (2003) desenvolveram um pão francês sem sal e relataram que na análise sensorial o pão foi aceito pela maioria dos provadores, com notas maiores que 6. Ignácio et al. (2013) em seus estudos afirmaram que os valores médios para os atributos sensoriais situaram-se entre 6,02 e 7,32 para os pães com até $1 \%$ de sal, ou seja, os provadores classificaram estes pães como "gostei ligeiramente" e "gostei moderadamente".
Apenas a formulação sem sal não foi tão bem aceita.

O teste para verificação da intenção de compra foi aplicado na amostra $\mathrm{C}$, devido conter farelo de trigo e menor quantidade de melhorador na sua formulação. Conforme a Figura 2, 46\% dos provadores certamente comprariam o produto e $36 \%$ provavelmente o comprariam.

Figura 2 - Intenção de compra do pão francês com redução do sal e farelo de trigo

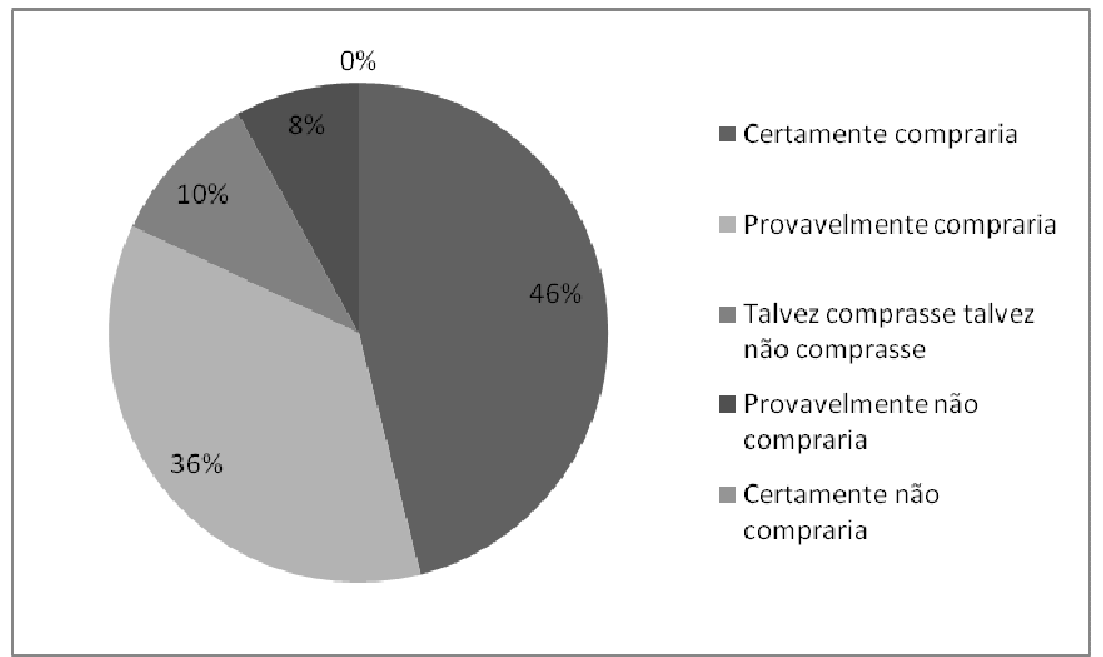

\section{CONCLUSÃO}

O consumo de produtos de panificação saudáveis ocasiona benefícios à saúde dos consumidores. A amostra com teor reduzido de sal e com fibras apresentou menor teor de sódio e maior teor de potássio e fibras. Os dados sensoriais deste trabalho permitem 
concluir que todas as amostras foram aceitas, sendo o pão com farelo de trigo e menor teor de sal e melhorador o de maior aceitabilidade.

\section{AGRADECIMENTOS}

Os autores agradecem ao Instituto Federal de Santa Catarina, Universidade Tecnológica Federal do Paraná e Fundação Araucária pelo apoio financeiro.

\section{REFERÊNCIAS}

ABIP. Disponível em <http://www.abip.org.br/noticias_internas.asp x?cod=164> . Acesso em 30 de abril de 2014 .

ADA. American Dietetic Association. (2002) "Position of the American Dietetic Association: health implications of dietary fiber." American Dietetic Association, v.102, p. 993-1000.

ANVISA. Agência Nacional de Vigilância Sanitária. Guia de boas práticas nutricionais pão francês. Disponível em:<http://portal.anvisa.gov.br/wps/wcm/con nect/9806518049af6bb196eab66dcbd9c63c/G uia+de+Boas+Pr\%C3\%A1ticas+Nutricionais + para + p\%C3\%A3o+franc\%C3\%AAs.pdf?M OD=AJPERES $>$. Acesso em 05/06/2013.
BORGES, J.T.da S.; PIROZI, M.R.; DE PAULA, C.D.; RAMOS, D.L.; CHAVES, J.B.P. Caracterização físico-química e sensorial de pão de sal enriquecido com farinha integral de linhaça. Boletim CEPPA, Curitiba, v. 29, n. 1, p. 83-96, jan./jun. 2011.

CAUVAIN, S. P. Reduced salt in bread and other baked products. Reducing Salt in Foods, part 3, 2007, p. 283-295.

DELLA TORRE, J. C. de M.; RODAS, M. A. de B.; BADOLATO, G. G.; TADINI, C. C. Perfil sensorial e aceitação de suco de laranja pasteurizado minimamente processado. Ciência e Tecnologia de Alimentos, v. 23, n. 2, p. 105-111, maio/ago. 2003.

DELCOUR, J.A.; HOSENEY, R.C. Principles of cereal science and technology. 3 ed., AACC International, St Paul, MN, USA, 2010. 261p.

HAGER, A. S.; WOLTER, A.; CZERNY, M.; BEZ, J.; ZANNINI, E.; ARENDT, E. K.; CZERNY, M. Investigation of product quality, sensory profile and ultrastructure of breads made from a range of commercial gluten-free flours compared to their wheat counterparts. Europe Food Research and Technology, v.235, p. 333-344, 2012. 
HALLÉN, E.; İBANOĞLU, Ş.; AINSWORTH, P. Effect of fermented/ germinated cowpea flour addition on the rheological and baking properties of wheat flour. Journal of Food Engineering, v. 63, p. 177-184, 2004.

IGNÁCIO, A.K.F.; RODRIGUES, J.T.de D.; NIIZU, P.Y.; CHANG, Y.K. Efeito da substituição de cloreto de sódio por cloreto de potássio em pão francês. Brazilian Journal of Food Science and Technology, v. 16, n. 1, p. 1-11, jan./mar. 2013.

INSTITUTO ADOLFO LUTZ. Normas analíticas do Instituto Adolfo Lutz. São Paulo: Instituto Adolfo Lutz, 1985.

LAI, C.S.; HOSENEY,R.C.; DAVIS,A.B. Effects of wheat bran in breadmaking. Cereal Chemistry, v.66, p.217-219, 1989.

MENDONÇA, H., SILVA, M.L. Redução da ingestão de sal como estratégia para a diminuição do risco cardiovascular. Revista Factores de Risco, n. 25, p. 24-26, 2012.

MEILGAARD, M.; CIVILLE, G. V.; CARR, B. T. Sensory Evaluation Techniques. 4 ed., Boca Raton, FL: CRC Press, 2007, 448p.

MINISTÉRIO DA SAÚDE. Termo de Compromisso entre o Ministério da Saúde e a Associação Brasileira das Indústrias de Alimentação (ABIA), Associação Brasileira das Indústrias de Massas Alimentícias (ABIMA), Associação Brasileira da Indústria de Trigo (ABITRIGO) e a Associação Brasileira da Indústria de Panificação e Confeitaria (ABIP) com a finalidade de estabelecer metas nacionais para redução do teor de sódio em alimentos processados no Brasil. Brasília - DF. 2011. Disponível em http://189.28.128.100/nutricao/docs/boletimSi svan/TERMO_DE_COMPROMISSO_2_SO DIO.pdf. Acesso em 20 de fevereiro de 2014.

ORNELLAS, L.H. Técnica dietética: seleção e preparo de alimentos. São Paulo: Ateneu; 2001. 142 p.

PHILIPPI, S. T.; SZARFARC, S. C.; LATTERZA, A. R. Virtual Nutri Software. v 1.0 for Windows. São Paulo: Departamento de Nutrição, Faculdade de Saúde Pública, Universidade de São Paulo, 1996.

PLESSAS, S.; TRANTALLIDI, M.; BEKATOROU, A.; KANELLAKI, M.; NIGAM, P.; KOUTINAS, A.A. Immobilization of kefir and Lactobacillus casei on brewery spent grains for use in sourdough wheat bread making. Food Chemistry, v. 105, p. 187-194, 2007. 
SILVA, M.E.M.P.e; YONAMINE; G.H.; VIEIRA, E; OLIVEIRA, B.M.P.M..; MITSUKI, L. Desenvolvimento e avaliação SOARES, M.E; PINHO, O. Estudo do teor de de pão francês caseiro sem sal. Brazilian sódio em pão consumido no Porto. Journal of Food Technology, v.6, n.2, p. Alimentação Humana, v.13, n.3, 2007. 229-236, jul./dez., 2003.

VIEGAS, C. Sal e doença cardiovascular.

Revista Factores de Risco, n. 10, p.12-18, Jul-Set, 2008. 